\title{
CORRESPONDANCE ENTRE DEUX SURFACES PAR QUATRE COUPLES DE TANGENTES PARALLELES
}

\author{
PAVEL DRĂGILĂ
}

1. La correspondance par parallélisme des plans tangents, aux points homologues des deux surfaces, fut étudié pour la première fois par Peterson. Il croyait avoir établi qu'en cas de cette correspondance, sur chaqune des surfaces il y en a toujours deux et seulement deux directions, qui sont parallèles aux directions correspondantes sur l'autre surface, et que de plus ces directions sont conjuguées sur les deux surfaces. Si nous désignons les deux surfaces par $S(x, y, z), \bar{S}(\bar{x}, \bar{y}, \bar{z})$ et les dérivées $\partial r / \partial u, \partial r / \partial v, \partial^{2} r / \partial u \partial v \ldots$ par les notations usuelles $r_{u}, r_{v}, r_{u v} \cdots$, la correspondance par parallélisme au sens de Peterson est définie par les relations

$$
\frac{\bar{x}_{u}}{x_{u}}=\frac{\bar{y}_{u}}{y_{u}}=\frac{\bar{z}_{u}}{z_{u}}=\lambda, \quad \frac{\bar{x}_{v}}{x_{v}}=\frac{\bar{y}_{v}}{y_{v}}=\frac{\bar{z}_{v}}{z_{v}}=\mu,
$$

c'est-à-dire

$$
\bar{r}_{u}=\lambda r_{u}, \quad \bar{r}_{v}=\mu r_{v},
$$

les paramètres curvilignes $u$, $v$ étant les mêmes sur les deux surfaces.

Nous avons démontré déjà qu'en déhors de ce cas de correspondance par parallélisme, il y a encore d'autres couples des surfaces parallèles, qui sont définies par les relations

$$
\bar{r}_{u}=\lambda r_{v}, \quad \bar{r}_{v}=\mu r_{u} .
$$

Il est aisé de vérifier que, si les coordonnées de la surface $\bar{S}$ satisfont une équation de Laplace

$$
\bar{r}_{u v}=a \bar{r}_{u}+b \bar{r}_{v},
$$

les coordonnées de l'autre surface $S$ vérifient le système d'équations

$$
\begin{aligned}
\mu r_{u u}+\mu_{u} r_{u} & =a \lambda r_{v}+b \mu r_{u}, \\
\lambda r_{v v}+\lambda_{v} r_{v} & =a \lambda r_{v}+b \mu r_{u},
\end{aligned}
$$

ce qui signifie qu'aux lignes $u, v$ conjuguées sur l'une des surfaces correspondent les lignes $u, v$ asymptotiques sur l'autre surface et réciproquement.

2. Je me suis proposé de chercher s'il y a des couples de deux surfaces se correspondant par plus que deux couples des tangentes

Received by the editors October 8, 1957. 
parallèles, dans leurs points homologues, et j'ai résolu affirmativement ce problème intéressant, au moyen des transformations (1).

Considérons à cet effet une surface $S$, dont les directions asymptotiques soient partout orthogonales. Il est bien connu que de telles surfaces existent, ces sont les surfaces minima. Sur ces surfaces, il est aisé de voir que, les directions asymptotiques sont les bissectrices des angles formés par les directions principales de la surface (la réciproque étant vraie pour toutes les surfaces). Supposons alors que la surface $S$ est rapportée aux lignes de courbure $u, v$ et cherchons ensuite la seconde surface $\bar{S}$, obtenue par les transformations (1). Cette nouvelle surface sera rapportée aux lignes asymptotiques $u, v$, qui sont rectangulaires, ce qui signifie qu'elle doit être aussi une surface minima. Les directions asymptotiques sur la première surface $S$ et les directions principles sur la seconde surface $\bar{S}$ doivent être aussi parallèles, étant les bissectrices des deux angles aux cotés parallèles.

Nous avons établi ainsi l'existence d'un nouveau cas de correspondance, entre deux surfaces, par quatre couples de tangentes parallèles, et découvert en même temps une transformation remarquable des surfaces minima.

3. Nous sommes ainsi arrivé au problème de la détermination des surfaces minima, qui soient rapportées aux lignes asymptotiques. Ce problème ne peut pas être résolu qu'en des cas particuliers.

Nous montrerons d'abord une propriété intéressante de ces surfaces que nous utilisons plus loin. Considérons à cette fin une surface quelconque, rapportée aux lignes asymptotiques $\alpha, \beta$ et supposons encore qu'elle soit satisfaite la relation

$$
E=G,
$$

où

$$
E=x_{\alpha}^{2}+y_{\alpha}^{2}+z_{\alpha}^{2} ; \quad G=x_{\beta}^{2}+y_{\beta}^{2}+z_{\beta}^{2} .
$$

Ecrivons ensuite l'équation des lignes de courbures

$$
\left|\begin{array}{cc}
E d \alpha+F d \beta & F d \alpha+G d \beta \\
D^{\prime} d \beta & D^{\prime} d \alpha
\end{array}\right|=0
$$

c'est-à-dire

$$
E\left(d \alpha^{2}-d \beta^{2}\right)=0 .
$$

Les directions principales sont données par les équations

$$
\alpha+\beta=2 u, \quad \alpha-\beta=2 v .
$$


Calculons ensuite les paramètres des directions asymptotiques

$$
r_{\alpha}=\frac{1}{2}\left(r_{u}+r_{v}\right), \quad r_{\beta}=\frac{1}{2}\left(r_{u}-r_{v}\right),
$$

et, en posant ensuite la condition d'orthogonalité

$$
r_{\alpha} r_{\beta}=0,
$$

nous obtenons la relation

$$
\sum r_{u}^{2}-\sum r_{v}^{2}=0
$$

ce que signifie que les lignes de courbures sont aussi isothèrmes.

4. Nous allons donner maintenant une méthode pour la détermination effective des couples de surfaces minima. Considérons pour cela deux fonctions $x(\alpha, \beta), y(\alpha, \beta)$, qui vérifient l'équation

$$
x_{\alpha} x_{\beta}+y_{\alpha} y_{\beta}=0 \text {. }
$$

On peut prendre alors pour coordonnées de la surfaces

$$
x=x(\alpha, \beta), \quad y=y(\alpha, \beta), \quad z=\beta .
$$

En posant encore la condition que les courbes $\alpha, \beta$ soient des asymptotiques, on aura les deux relations

$$
\begin{aligned}
& x_{\alpha \alpha} y_{\alpha}-y_{\alpha \alpha} x_{\alpha}=0, \\
& x_{\beta \beta} y_{\alpha}-y_{\beta \beta} x_{\alpha}=0 .
\end{aligned}
$$

Par l'intégration des deux équations (4) et (3) nous trouvons

$$
y_{\alpha}=h(\beta) x_{\alpha}, \quad x_{\beta}=-h(\beta) y_{\beta} .
$$

Posant encore la condition d'isothermie (2)

$$
x_{\alpha}^{2}+y_{\alpha}^{2}=x_{\beta}^{2}+y_{\beta}^{2}+1,
$$

nous obtenons la relation

$$
\left(1+h^{2}\right) x_{\alpha}^{2}=\left(1+h^{2}\right) y_{\beta}^{2}+1,
$$

qui est vérifie par les coordonnées de la surface

$$
\begin{aligned}
& x=\operatorname{sh} \alpha \cos \beta, \\
& y=\operatorname{sh} \alpha \sin \beta, \\
& z=\beta .
\end{aligned}
$$


C'est l'helicoïde gauche, rapportée aux lignes asymptotiques, pour laquelle on aura

$$
E=c h^{2} \alpha, \quad F=0, \quad G=1+s^{2} \alpha .
$$

Les lignes de courbures sont définies par l'équation

$$
d \alpha^{2}-d \beta^{2}=0,
$$

d'où il résulte

$$
\alpha+\beta=2 u, \quad \alpha-\beta=2 v .
$$

On peut alors écrire les coordonnées de la même surface, rapportée aux lignes de courbures

$$
\begin{aligned}
& x=\operatorname{sh}(u+v) \cos (u-v), \\
& y=\operatorname{sh}(u+v) \sin (u-v), \\
& z=u-v .
\end{aligned}
$$

En faisant ensuite les substitutions dans l'équation (1), il vient

$$
\begin{aligned}
& \bar{x}_{u}=\lambda[\operatorname{ch}(u+v) \cos (u-v)+\operatorname{sh}(u+v) \sin (u-v)], \\
& \bar{x}_{v}=\mu[\operatorname{ch}(u+v) \cos (u-v)-\operatorname{sh}(u+v) \sin (u-v)], \\
& \bar{y}_{u}=\lambda[\operatorname{ch}(u+v) \sin (u-v)-\operatorname{sh}(u+v) \cos (u-v)], \\
& \bar{y}_{v}=\mu[\operatorname{ch}(u+v) \sin (u-v)+\operatorname{sh}(u+v) \cos (u-v)], \\
& \bar{z}_{u}=-\lambda, \\
& \bar{z}_{v}=\mu .
\end{aligned}
$$

Des relations de condition

$$
\left(\bar{r}_{u}\right)_{v}=\left(\bar{r}_{v}\right)_{u}
$$

nous obtenons les deux équations

$$
\begin{aligned}
\lambda \sin (u-v)+\lambda_{v} \cos (u-v) & =-\mu \sin (u-v), \\
-\lambda \cos (u-v)+\lambda_{v} \sin (u-v) & =\mu \cos (u-v),
\end{aligned}
$$

d'où il résulte

$$
\lambda_{v}=0, \quad \mu_{u}=0,
$$

et enfin

$$
\lambda=-\mu=\text { const. }
$$

En considérant que les transformations homothétiques ne présentent pas d'importance dans ce problème, nous pourrons prendre

$$
\lambda=1, \quad \mu=-1,
$$


et nous obtenons ainsi la nouvelle surface $\bar{S}$, rapportée aux lignes asymptotiques $u, v$,

$$
\begin{aligned}
& \bar{x}=\operatorname{ch}(u+v) \sin (u-v), \\
& \bar{y}=\operatorname{ch}(u+v) \cos (u-v), \\
& \bar{z}=-u-v .
\end{aligned}
$$

Il est visible que nous avons obtenu une surface minima de révolution; il est très probable que c'est la seule surface de cet espèce.

Nous pouvons encore remarquer que les coefficients $\lambda, \mu$ doivent être liées par l'une des deux relations

$$
\lambda=\mu, \quad \lambda=-\mu .
$$

Il s'ensuit qu'on peut déterminer des relations (1) une (ou eventuellement deux) expression de $\lambda$ et $\mu$. Cela signifie qu'à une surface donnée $S$, correspond une ou bien deux surfaces transformées $\bar{S}$.

5. Il est manifeste que ce problème de la correspondance par plans tangents parallèles des deux surfaces, est étroitement lié à l'un des problèmes centraux de la Géométrie différentielle projective: la correspondance ponctuelle des deux surfaces (par des tangentes incidentes). Nous montrerons, dans un prochain travail, qu'il est possible de trouver un grand nombre de résultats nouveaux encore dans cette direction.

\section{BiBLIOGRAPHIE}

1. P. Drăgilă, Sur la correspondance par parallélisme de deux surfaces, Proc. Amer. Math. Soc. vol. 9 (1958) pp. 189-200.

Timisoara, Roumania 